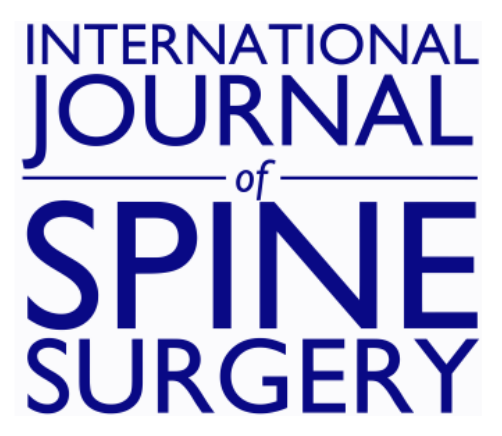

\title{
Does Resection of the Posterior Longitudinal Ligament Affect the Stability of Cervical Disc Arthroplasty?
}

\author{
LEONARD I. VORONOV, ROBERT M. HAVEY, PARMENION P. TSITSOPOULOS, SAEED \\ KHAYATZADEH, JEREMY GOODSITT, GERARD CARANDANG, ALEXANDER J \\ GHANAYEM and AVINASH G. PATWARDHAN
}

Int J Spine Surg 2018, 12 (2) 285-294

doi: https://doi.org/10.14444/5035

http://ijssurgery.com/content/12/2/285

This information is current as of April 26, 2023.

Email Alerts Receive free email-alerts when new articles cite this article. Sign up at: http://ijssurgery.com/alerts 


\title{
Does Resection of the Posterior Longitudinal Ligament Affect the Stability of Cervical Disc Arthroplasty?
}

\author{
LEONARD I. VORONOV, MD, PHD,,${ }^{1,2}$ ROBERT M. HAVEY, MS, ${ }^{1,2}$ PARMENION P. TSITSOPOULOS, MD, \\ PHD,${ }^{1}$ SAEED KHAYATZADEH, MS,${ }^{1}$ JEREMY GOODSITT, PhD,${ }^{1}$ GERARD CARANDANG, MS,${ }^{1}$ \\ ALEXANDER J. GHANAYEM, MD, ${ }^{1,2}$ AVINASH G. PATWARDHAN, PHD ${ }^{1,2}$ \\ ${ }^{I}$ Musculoskeletal Biomechanics Laboratory, Edward Hines Jr. VA Hospital, Hines, Illinois, ${ }^{2}$ Department of Orthopaedic Surgery and Rehabilitation, Loyola \\ University Medical Center, Maywood, Illinois
}

\begin{abstract}
Background: The need for posterior longitudinal ligament (PLL) resection during cervical total disc arthroplasty (TDA) has been debated. The purpose of this laboratory study was to investigate the effect of PLL resection on cervical kinematics after TDA.

Methods: Eight cadaveric cervical spine specimens were tested in flexion-extension (FE), lateral bending (LB), and axial rotation (AR) to moments of $\pm 1.5 \mathrm{Nm}$. After testing the intact condition, anterior C5-C6 cervical discectomy was performed followed by PLL resection and implantation of a compressible, 6-degrees-of-freedom disc prosthesis (M6-C, Spinal Kinetics Inc, Sunnyvale, California). Next, a second prosthesis was implanted at C6-C7 with PLL intact. Finally, the C6-C7 PLL was resected while the disc prosthesis remained in place. Segmental range of motion (ROM) and stiffness in the high flexibility zone around the neutral posture were analyzed using repeated measures ANOVA.

Results: At C5-C6, following TDA and PLL resection, FE, LB, and AR ROMs decreased significantly. Anterior and posterior disc height, segmental lordosis, and flexion stiffness increased significantly. At C6-C7, TDA with the PLL intact resulted in a significant increase in anterior disc height and segmental lordosis with no change in posterior disc height. FE, LB, and AR ROMs all decreased significantly, while flexion stiffness increased significantly compared to intact. PLL resection at C6-C7 did not result in a notable change compared to TDA with PLL intact. At the same level, flexion stiffness decreased following PLL resection compared to TDA with a value closer to intact. Two-level TDA (C5C7) with PLL resection did not result in a loss of segmental stability.

Conclusion: PLL resection did not significantly affect motion segment kinematics following cervical TDA using a prosthesis with inherent stiffness. Motion segment stiffness loss after PLL resection can be compensated for by a TDA design that can provide resistance to angular motion.

Biomechanics

Keywords: cervical spine, total disc arthroplasty, posterior longitudinal ligament, stability, ligament resection
\end{abstract}

\section{INTRODUCTION}

Total disc arthroplasty (TDA) has been shown to be a viable alternative to fusion in treating a subset of patients with symptomatic cervical radiculopathy and myelopathy. ${ }^{1-4}$ During TDA implantation, the structural integrity of the motion segment is compromised due to soft tissue resection and neural decompression. During decompression, the posterolateral aspects of the annulus and posterior longitudinal ligament (PLL) are resected to decompress the nerve root, and, if necessary, the central portion of the PLL is resected to decompress the spinal cord. ${ }^{1,5,6}$ It is commonly believed that the functional PLL (fPLL), which includes the multiple layers of the ligament and thickened posterior annulus fibrosis (Figure 1), provides stability to cervical motion segments. However, PLL resection during fusion and disc arthroplasty has long been debated with numerous conflicting references explaining the role and strength of the PLL. ${ }^{7-12}$

Akaishi concluded that the PLL provides more stability than the anterior longitudinal ligament because of its higher dynamic stiffness. ${ }^{13}$ Others suggested that PLL is thinner and weaker than the anterior longitudinal ligament $\mathrm{t}^{12,14-16}$ with proponents of its resection suggesting that it plays only a minor role in motion segment stability compared to the remaining ligamentous structures. ${ }^{17-19}$ During TDA, PLL resection is suggested to facilitate a more parallel distraction, better decompression, and disc height restoration and ensures segment mobility. ${ }^{5,20-22}$ On the contrary, it has been postulated 

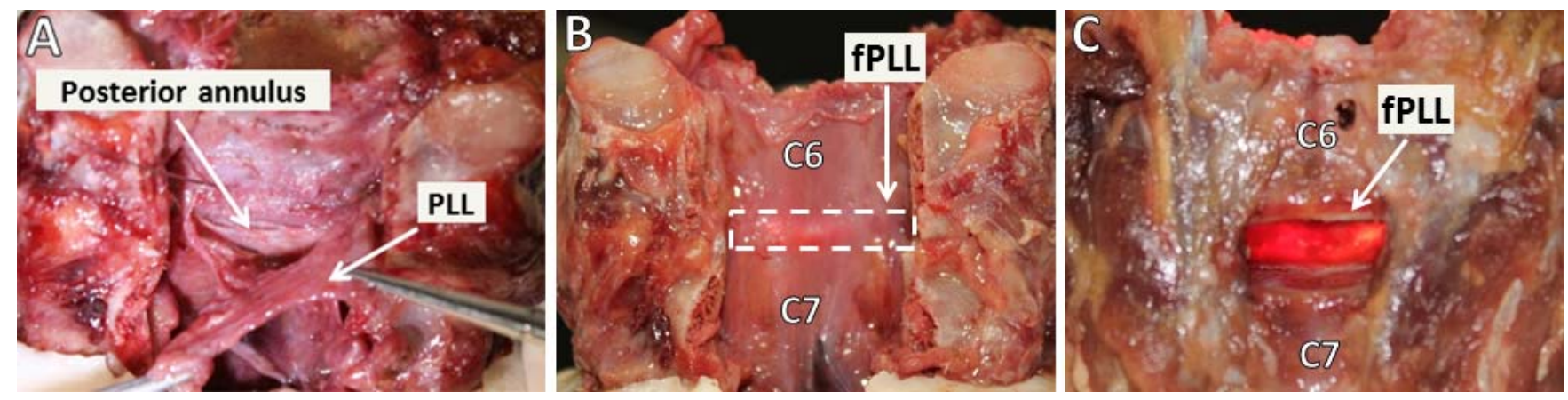

Figure 1. Motion segment with lamina removed. (A) Posterior view of functional posterior longitudinal ligament (fPLL); includes thickened posterior annulus fibrosis and posterior longitudinal ligament. (B) Posterior to anterior view. (C) Anterior to posterior view.

that PLL should not be excised in order to maintain its stabilizing effect. ${ }^{12,13,23}$

In order to add stability after PLL resection, it has been suggested that a taller artificial disc could counter the destabilization effect of PLL resection by relying on ligament tension to provide motion segment stiffness. ${ }^{24}$ However, ligament tensioning through the use of segment distraction can have potential negative effects, such as facet joint subluxation, failure of the tensioned ligaments, and altered segmental biomechanics, if height is increased beyond that of the native disc. ${ }^{24} \mathrm{~A}$ possible alternative is to utilize the mechanics of the TDA prosthesis to provide segmental stiffness. Several TDA devices have inherent stiffness due to their designs. To our knowledge, biomechanical data supporting their ability to offset instability introduced by PLL resection in cervical TDA constructs do not exist.

The purpose of this study was to determine the effects of PLL resection on the kinematic behavior of the cervical spine using an artificial disc that offers resistance to angular and translation motions. Specifically, the hypothesis that implantation of a cervical artificial disc with mechanical stiffness will compensate for the instability caused by PLL resection during 1-level and 2-level TDA was tested.

\section{MATERIALS AND METHODS}

\section{Specimens and Experimental Setup}

Eight fresh-frozen cadaveric subaxial cervical spines (C3-T1) with mean age of $43 \pm 7.9$ years old, 6 male and 2 female, were tested. Radiographs were taken to exclude severe degeneration, trauma, tumors, or other conditions that could significantly impact the natural kinematics of the specimen. The specimens were sealed in plastic bags and frozen at $-20^{\circ} \mathrm{C}$ prior to testing. Specimens were thawed and cleaned the day of testing. Muscles were removed, preserving the osteoligamentous structures important for stability.

Five radiopaque fiduciary markers were rigidly attached to each vertebral body prior to computed tomography $(\mathrm{CT})$ imaging. After obtaining an axial fine-slice $(0.63 \mathrm{~mm})$ CT scan (LightSpeed VPT, GE Medical Systems, Salt Lake City, Utah), the 3dimensional (3D) geometry of each vertebral body was reconstructed individually using Mimics software (Materialise, Inc, Plymouth, Michigan).

Each specimen was anchored in aluminum cups using k-wires and bone cement at the $\mathrm{C} 3$ and $\mathrm{T} 1$ vertebrae. Specimens were then mounted on a 6component load cell (Model MC3A-6-250, AMTI multicomponent transducers, AMTI Inc, Newton, Massachusetts) and allowed to move freely at the proximal end. This setup allowed for unconstrained continued cycling of the specimen between specified moment end points in flexion-extension (FE), lateral bending (LB), and axial rotation (AR). AR was performed using a force couple to apply a pure moment, while FE and LB were performed using a variable force at a distance. The moment arm length was $50 \mathrm{~cm}$ for LB and $60 \mathrm{~cm}$ for FE. The experiment was performed at room temperature. Tissue hydration was maintained by wrapping the specimens in saline-soaked gauze.

A compressive follower preload of $150 \mathrm{~N}$ was applied to the specimen along an optimized path during FE testing. Follower preload is a technique used in the laboratory to approximate compressive loads to a multisegment spine. This technique mimics the action of the muscles in vivo responsible for maintaining head posture. The preload was applied along the cervical spine's lordotic curve in order to allow the specimen to support the physiologic load without limiting the specimen's range of motion. ${ }^{25,26}$ 


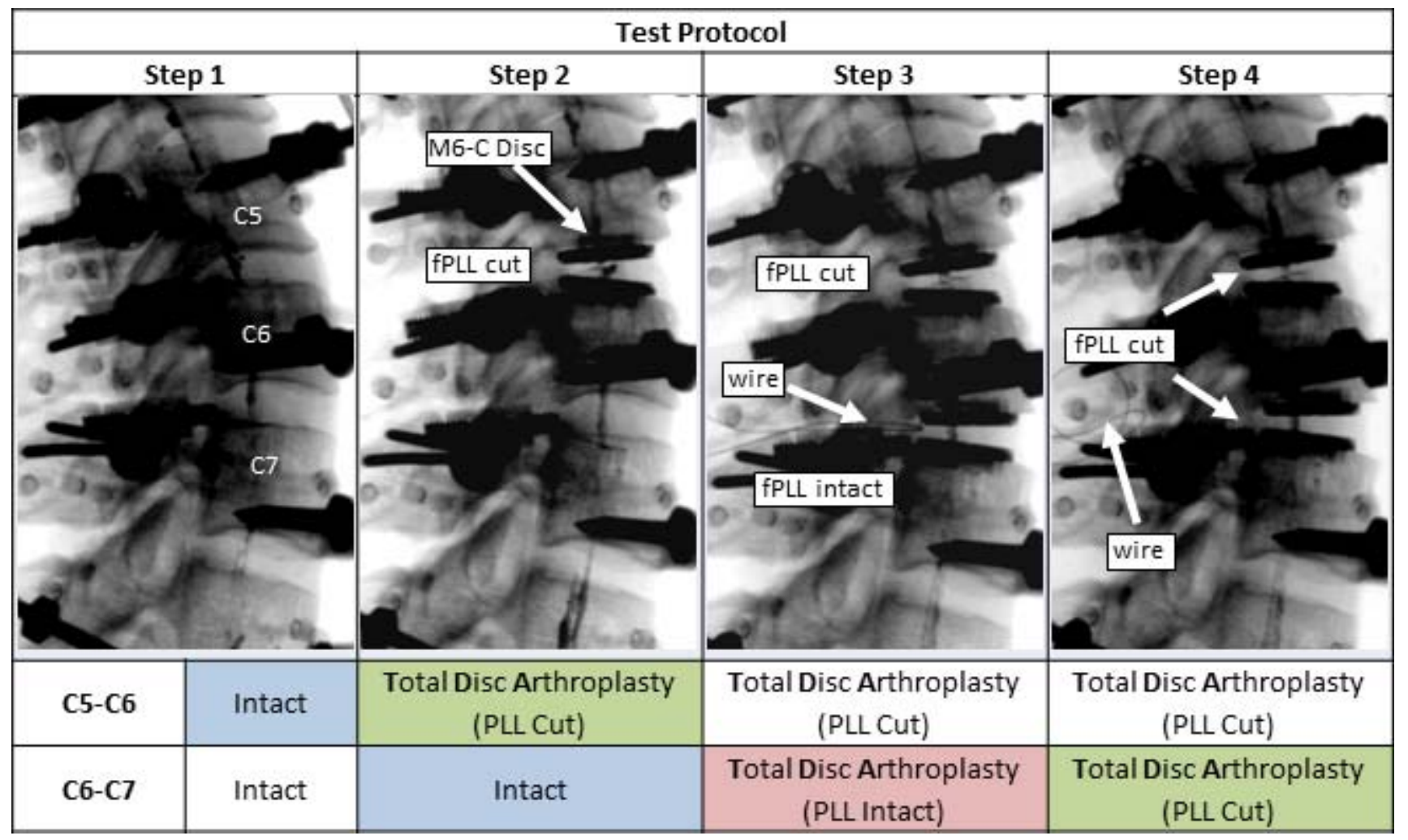

Figure 2. Test protocol. Flexion-extension, lateral bending, and axial rotation tests $( \pm 1.5 \mathrm{Nm})$ were performed at all protocol steps. Abbreviations: PLL, posterior longitudinal ligament; fPLL, functional PLL.

Prior to kinematic testing, the locations of the fiducial markers were digitized in relation to the motion measurement targets attached to each vertebral body. The motions of C3, C4, C5, C6, and $\mathrm{C} 7$ levels were measured in 3 dimensions relative to the stationary $\mathrm{T} 1$ vertebra attached to the base of the kinematic testing apparatus. Vertebral motions were measured using an optoelectronic motion measurement system (Optotrak Certus, Northern Digital Inc, Waterloo, Ontario, Canada).

The specimen-specific CT-based kinematic analysis was performed for each specimen individually by combining the motion data collected optoelectronically with the CT scan-based 3D geometry data. This provided the ability of accurately measuring anatomical parameters, such as segmental wedge angles (segmental lordosis) and disc height, during testing. The mathematical combination of the specimen motion and 3D geometry data sets was feasible by using the location of radiopaque fiducial markers known in both data sets. This method has been previously described in Havey et al. $^{27}$

\section{Experimental Protocol}

Baseline range of motion ( $\mathrm{ROM}$ ) was determined in FE, LB, and AR in load-control mode under $\pm 1.5-\mathrm{Nm}$ bending moments without preload. FE was also tested with a follower preload of $150 \mathrm{~N}$. Load-displacement data were collected until 2 reproducible load displacement loops were obtained.

After testing the intact spine (Figure 2, step 1), anterior cervical discectomy with narrow window and PLL resection was performed at the C5-C6 level. The end plates were prepared by removing the cartilage to expose cortical bone while leaving the uncinate processes intact. This was followed by prosthesis implantation (M6-C, Spinal Kinetics Inc, Sunnyvale, California) according to manufacturer guidelines.

ROM testing was repeated (Figure 2, step 2). Next, an additional prosthesis was implanted at C6$\mathrm{C} 7$ following anterior discectomy with the prosthesis again positioned posterior to the midline of the disc, leaving the uncinate processes and PLL intact (Figure 2, step 3). Finally, the functional PLL was resected at $\mathrm{C} 6-\mathrm{C} 7$ while keeping the disc prosthesis in place (Figure 2, step 4). 

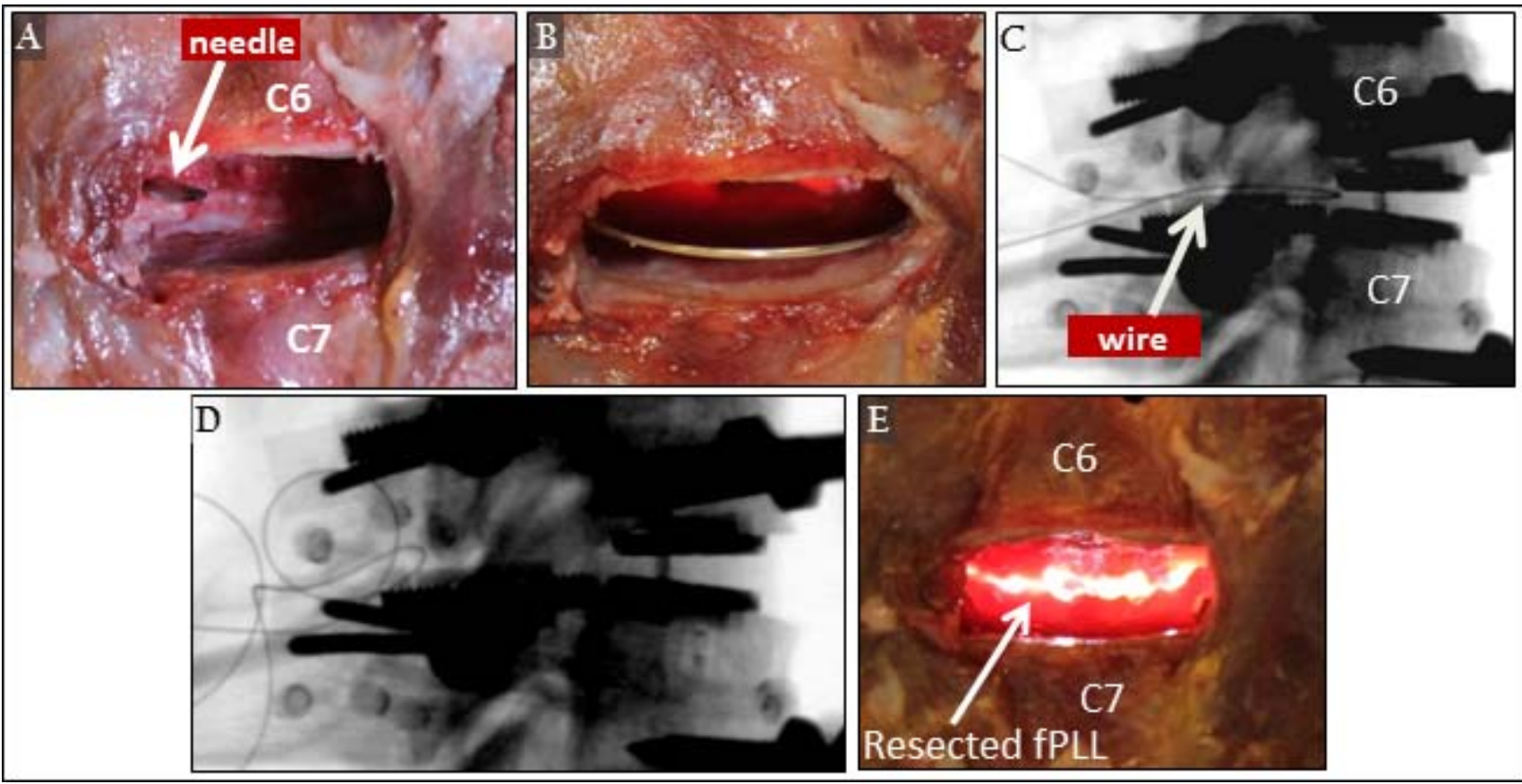

Figure 3. Technique to resect functional posterior longitudinal ligament (PLL) without prosthesis removal. (A) Needle insertion. (B) Wire inserted through ligamentum flavum, looped around functional PLL (fPLL), and passed back through needle. (C) Wire around PLL with total disc arthroplasty in place. (D) After PLL is cut. (E) Posttest confirmation of PLL resection.

Resection of the PLL at C6-C7 without removing the TDA was accomplished by placement of a 0.36mm stainless-steel wire looped around PLL. Placement of this wire was done after performing the discectomy but prior to TDA insertion. The wire was introduced posteriorly through a single 16gauge needle puncture hole in the ligamentum flavum (Figure 3A through $\mathrm{C}$ ) to minimize soft tissue disruption that could affect segmental kinematics. After kinematic testing of the specimen with 2-level TDA, the wire was used to cut through the function PLL. Fluoroscopy was used to verify successful transection of the PLL by observing when the stainless-steel wire had passed into the canal space (Figure 3D). The flexibility tests were repeated after PLL resection. Once kinematic testing was completed, the posterior spinal elements were removed to confirm complete PLL resection (Figure 3E).

\section{Kinematic and Statistical Analysis}

Stability comparisons were evaluated based on ROM of each motion segment as well as the shape of the flexibility curve. ROM and stiffness were derived from load vs. angular motion data. Measurements of ROM and stiffness are described in Figure 4A. These were analyzed using repeated measures ANOVA and post hoc testing using the
Holm-Bonferroni correction for multiple comparisons. ${ }^{28}$ The level of significance was set at $P<.05$. Data were analyzed for FE with $150 \mathrm{~N}$ follower preload and for LB and AR with no preload. The statistical software Systat (version 10.2, Richmond, California) was used for analysis.

\section{RESULTS}

\section{Disc Height}

Segmental disc height and wedge angle were measured using the 3D specimen-specific kinematic methodology. At C5-C6, following TDA and PLL resection, the change in anterior and posterior disc height from intact to TDA at $\mathrm{C} 5-\mathrm{C} 6$ was $+2.2 \pm 0.7 \mathrm{~mm}$ and $+1.6 \pm 1.0 \mathrm{~mm}(P<.01)$, respectively (Table 1, Figure 5). This resulted in an increase in segmental lordosis of $2.8 \pm 3.5$ degrees $(P<.05)$.

At C6-C7, the intact condition was initially compared to TDA with the PLL intact. The change in anterior and posterior disc height from intact to TDA was $+1.7 \pm 1.0 \mathrm{~mm}(P<.01)$ and $+0.5 \pm 1.1$ $\mathrm{mm}(P=.2)$, with a resulting increase in segmental lordosis of $+5.4 \pm 2.8$ degrees $(P<.01)$. With TDA in situ, subsequent PLL resection at C6-C7 resulted in no significant change in disc height or segmental lordosis $(P>.05$; Table 2$)$. 

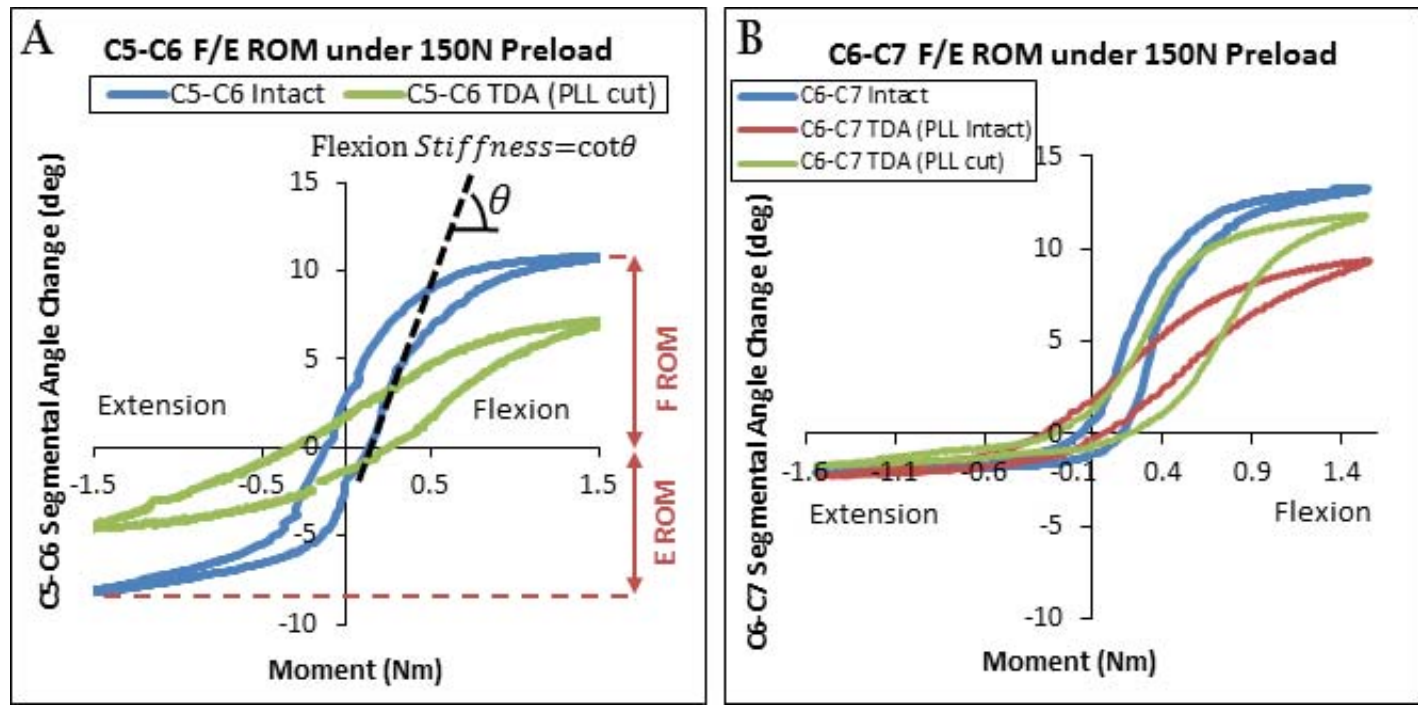

Figure 4. Flexion-extension (FE) load displacement of C5-C6 (A) and C6-C7 (B) segments at all protocol steps demonstrating range of motion (ROM) and segmental stiffness. Abbreviations: TDA, total disc arthroplasty; PLL, posterior longitudinal ligament.

\section{ROM}

At C5-C6, FE, LB, and AR ROM all decreased significantly after TDA compared to the intact condition (Figures 4A and 6A). When evaluating flexion ROM (F-ROM) and extension ROM (EROM) separately, a significant reduction in both (FROM: $8.8 \pm 3.4$ to $5.1 \pm 2.1$ degrees, $P<.05$; EROM: $6.5 \pm 2.5$ to $3.5 \pm 1.7$ degrees, $P<.01)$ was noted (Figure 6B).

For the C6-C7 motion segment, TDA without PLL resection resulted in a significant decrease in FE ROM (FE-ROM) compared to intact $(13.0 \pm 3.4$ to $9.5 \pm 2.5$ degrees; $P<.01)$. After PLL resection, FE-ROM increased slightly $(9.5 \pm 2.5$ to $10.6 \pm 2.5$ degrees, $P=.08)$ but still lower than intact $(10.6 \pm 2.5$ vs. $13.0 \pm 3.4$ degrees, $P=.05$ ) (Figures 4B and 6C). F-ROM decreased significantly after TDA compared to intact without PLL resection $(8.2 \pm 3.2$ to $6.2 \pm 2.6$ degrees, $P<.05)$. Following PLL resection, F-ROM increased to $7.2 \pm 2.9$ degrees $(P=.08)$ but remained less than intact $(7.2 \pm 2.9$ vs. $8.2 \pm 3.2$ degrees, $P=.10)$. E-ROM was not affected by TDA or PLL resection $(P=.11$ and $P=.77)$ (Figure 6D). AR and LB ROM were significantly affected by TDA with intact PLL $(P<.01)$ (Figure 6C), and resection of the PLL had no additional effect.

\section{Stiffness}

At $\mathrm{C} 5-\mathrm{C} 6$, the reduction in $\mathrm{ROM}$ following TDA with the PLL resected resulted in significantly increased stiffness in flexion and extension $(0.08 \pm 0.09$ to $0.21 \pm 0.13 \mathrm{Nm} /$ degree, $P<.05)$ (Figure 7).

At C6-C7, segmental flexion stiffness significantly increased after TDA with intact PLL $(0.09 \pm 0.05$ vs. $0.18 \pm 0.08 \mathrm{Nm} /$ degree, $P<.05)$. After PLL resection, motion segment stiffness decreased significantly compared to intact PLL $(0.13 \pm 0.06$ $\mathrm{Nm} /$ degree, $P<.05)$. This value was closer to intact stiffness and significantly different than the stiffness with intact PLL (Figure 7A).

Table 1. C5-C6 disc height and wedge angle for each protocol step with comparison to Lauryssen s in vivo feasibility study.

\begin{tabular}{lll}
\hline & \multicolumn{2}{c}{ Surgical Steps } \\
\cline { 2 - 3 } & \multicolumn{1}{c}{ Intact } & TDA (PLL Cut) \\
\hline This study, in vitro, C5-C6 $(\mathrm{n}=8)$ & & \\
C5-C6 anterior disc height & $4.2 \pm 1.3 \mathrm{~mm}$ & $6.5 \pm 0.9 \mathrm{~mm}$ \\
C5-C6 posterior disc height & $2.5 \pm 1.2 \mathrm{~mm}$ & $4.2 \pm 1.1 \mathrm{~mm}$ \\
C5-C6 wedge angle & $6.5 \pm 4.5$ degrees & $9.3 \pm 2.7$ degrees \\
Lauryssen, in vivo, 30 patients, 36 levels, C3-T1, 21 to 75 years old & & \\
Anterior disc height & $4.2 \mathrm{~mm}$ & $6.8 \mathrm{~mm}$ \\
Posterior disc height & $3.3 \mathrm{~mm}$ & $5.1 \mathrm{~mm}$ \\
Wedge angle & 2.8 degrees & 5.8 degrees \\
\hline
\end{tabular}

Abbreviations: TDA, total disc arthroplasty; PLL, posterior longitudinal ligament. 


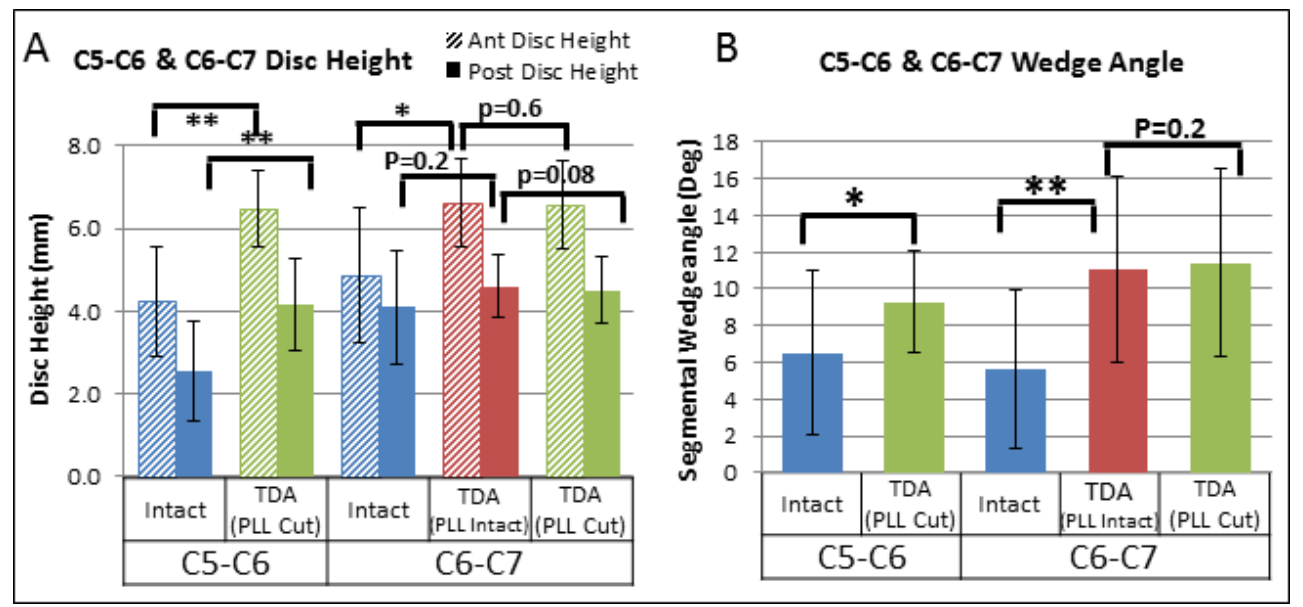

Figure 5. (A) $\mathrm{C} 5-\mathrm{C} 6$ and $\mathrm{C} 6-\mathrm{C} 7$ anterior and posterior disc heights for all protocol steps. (B) C5-C6 and $\mathrm{C} 6-\mathrm{C} 7$ segmental wedge angles for all protocol steps. $\left.{ }^{*} P \leq .05,{ }^{* *} P \leq .01\right)$. Abbreviations: TDA, total disc arthroplasty; PLL, posterior longitudinal ligament.

Two-level TDA (C5-C7) with PLL resection did not result in instability. Two-level stiffness increased insignificantly compared to intact (Table 3). Noticeably, this change in stiffness resulted in a decrease in ROM compared to intact $(P<.01)$ (Figure 7B).
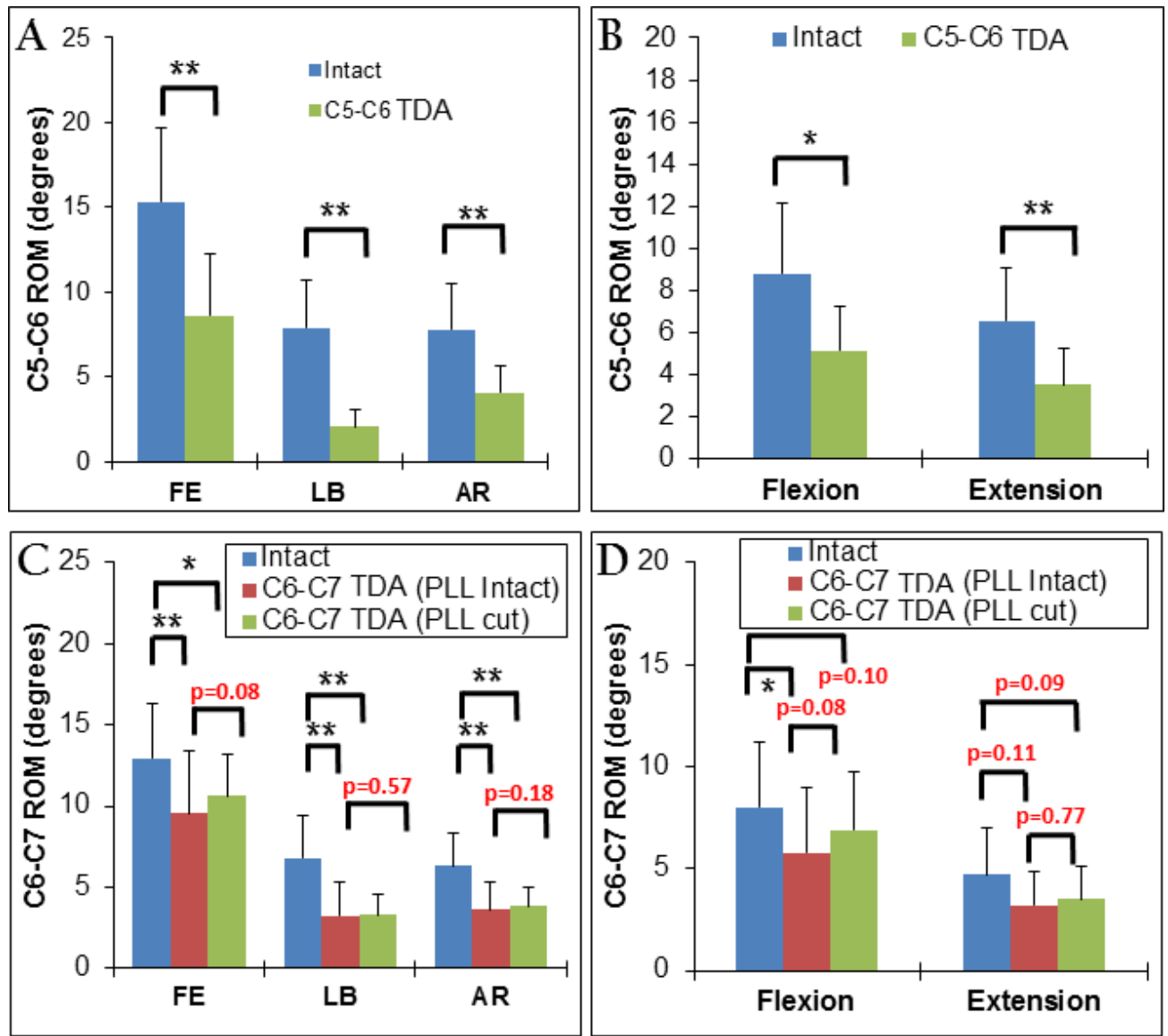

Figure 6. Range of motion (ROM) for all protocol steps: (A) C5-C6 ROM in flexion-extension (FE), lateral bending (LB), and axial rotation (AR) tests. (B) C6-C7 ROM in FE, LB, and AR tests. (C) C5-C6 ROM in flexion and extension. (D) C6-C7 ROM in flexion and extension. ${ }^{*} P \leq .05$, ${ }^{* *} P \leq .01$. Abbreviations: TDA, total disc arthroplasty; PLL, posterior longitudinal ligament. 


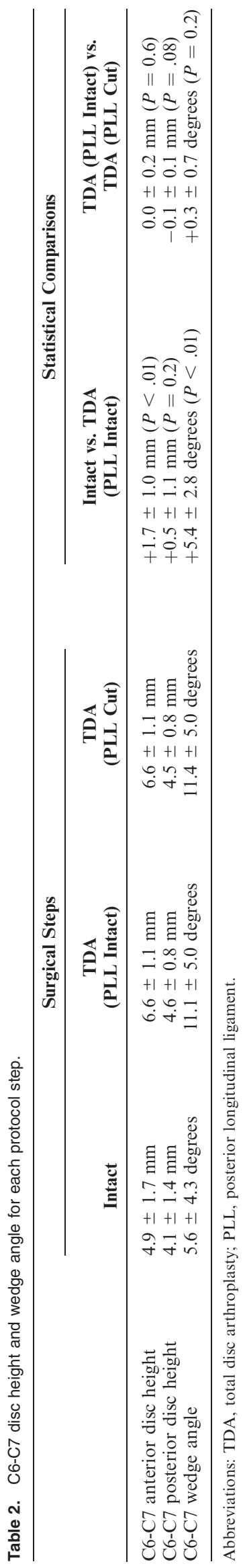

comes. $^{2,20,22,29,30}$ Many surgeons routinely remove the PLL during decompression, ${ }^{1-3,5}$ while others recommend removal only in cases where the PLL directly contributes to neurologic compression or when there are disc fragments behind the PLL. ${ }^{12,17,31,32}$ Disc space distraction caused by prosthesis insertion tensions the remaining soft tissue structures, potentially compensating for PLL resection. $^{2}$ Interestingly, some authors suggest preservation of the PLL for stability, depending mainly on the implant design. ${ }^{12,24,33}$

In cadaveric studies, Chen et al. ${ }^{17}$ found a significant reduction in motion segment stiffness after PLL resection, McAfee et al. ${ }^{12}$ and Cunningham et al. ${ }^{20}$ showed increased ROM after arthroplasty with PLL resection. ${ }^{1,34}$ It has been argued that retaining the PLL may help prevent hyperflexion and overdistraction of the disc space and facet joints, decreasing the incidence of iatrogenic injury, such as dural tears. ${ }^{6,11,13,14}$

Our hypothesis suggested that resection of the supportive soft tissues, such as the anterior longitudinal ligament, annulus, and PLL during decompression and TDA placement, transfers the burden of stability onto the prosthesis and remaining soft tissue structures. By using an artificial disc that has inherent stiffness, the 1-level TDA at C5-C6 was expected to experience equivalent or decreased ROM compared to the intact condition in all 3 motion planes (FE, LB, and AR) with a resulting increase in motion segment stiffness. This was confirmed for C5-C6 TDA with PLL resection since segmental stiffness in the high-flexibility zone increased.

Surgery at C6-C7 was performed in 2 steps, allowing a more in-depth analysis of PLL resection. In the first step, leaving the PLL intact resulted in higher flexion stiffness and decreased ROM compared to intact. Following PLL resection (step 2), ROM increased but remained significantly smaller than intact, while stiffness returned closer to that of the intact motion segment.

The sequence of PLL resection had an effect on sagittal balance. Segmental lordosis and anterior disc space height were both significantly different after TDA regardless of PLL resection for both operative levels (C5-C6 and C6-C7). Cutting PLL prior to TDA implantation at C5-C6 allowed better maintenance of neutral posture. TDA at C6-C7 with intact PLL resulted in a much greater increase in 
Table 3. Range of motion (ROM) and stiffness properties of $\mathrm{C} 5-\mathrm{C} 7$ for all protocol steps.

\begin{tabular}{|c|c|c|c|c|c|c|c|}
\hline $\begin{array}{l}\text { Motion } \\
\text { Segment }\end{array}$ & Protocol Step & $\begin{array}{c}\text { FE-ROM, } \\
\text { degrees }\end{array}$ & $\begin{array}{c}\text { F-ROM, } \\
\text { degrees }\end{array}$ & $\begin{array}{c}\text { E-ROM, } \\
\text { degrees }\end{array}$ & $\begin{array}{c}\text { LB-ROM, } \\
\text { degrees }\end{array}$ & $\begin{array}{c}\text { AR-ROM, } \\
\text { degrees }\end{array}$ & $\begin{array}{l}\text { F-Stiffness, } \\
\text { Nm/degree }\end{array}$ \\
\hline \multirow[t]{2}{*}{$\mathrm{C} 5-\mathrm{C} 6$} & Step 1 (intact) & $15.3 \pm 4.3$ & $8.8 \pm 3.4$ & $6.5 \pm 2.5$ & $7.9 \pm 2.9$ & $7.8 \pm 2.7$ & $0.08 \pm 0.06$ \\
\hline & Step 2 (TDA with PLL cut) & $8.6 \pm 3.6$ & $5.1 \pm 2.1$ & $3.5 \pm 1.7$ & $2.1 \pm 1.0$ & $4.1 \pm 1.6$ & $0.21 \pm 0.11$ \\
\hline \multirow[t]{3}{*}{$\mathrm{C} 6-\mathrm{C} 7$} & Step 2 (intact) & $13.0 \pm 3.4$ & $8.0 \pm 3.2$ & $4.7 \pm 2.3$ & $6.8 \pm 2.6$ & $6.3 \pm 2.0$ & $0.09 \pm 0.05$ \\
\hline & Step 3 (TDA with PLL intact) & $9.5 \pm 2.5$ & $5.8 \pm 2.6$ & $3.2 \pm 1.1$ & $3.2 \pm 1.2$ & $3.6 \pm 1.0$ & $0.18 \pm 0.08$ \\
\hline & Step 4 (TDA with PLL cut) & $10.6 \pm 3.4$ & $6.9 \pm 2.8$ & $3.5 \pm 1.6$ & $3.3 \pm 1.3$ & $3.8 \pm 1.1$ & $0.13 \pm 0.06$ \\
\hline \multirow[t]{3}{*}{$\mathrm{C} 5-\mathrm{C} 7$} & Step 1 (intact) & $27.3 \pm 7.1$ & $\mathrm{~N} / \mathrm{A}$ & & & & $0.18 \pm 0.12$ \\
\hline & Step 2 (1-level TDA with PLL cut) & $21.0 \pm 6.0$ & & & & & $0.31 \pm 0.14$ \\
\hline & Step 4 (2-level TDA with PLL cut) & $19.3 \pm 5.9$ & & & & & $0.34 \pm 0.17$ \\
\hline
\end{tabular}

Abbreviations: FE-ROM, flexion-extension range of motion; F-ROM, flexion ROM; E-ROM, extension-ROM; LB-ROM, lateral bending ROM; AR-ROM, axial rotation ROM; F-stiffness, flexion stiffness; TDA, total disc arthroplasty; PLL, posterior longitudinal ligament.

segmental lordosis than TDA at C5-C6 with PLL cut.

Posterior disc height at C5-C6 increased significantly after TDA, while the posterior disc height at C6-C7 did not change significantly after TDA with intact PLL. Interestingly, the posterior height at C5C6 with TDA and PLL cut was equivalent to the posterior height at $\mathrm{C} 6-\mathrm{C} 7$ in the intact condition, and posterior disc height did not change after TDA at C6-C7 with or without PLL cut. Further, PLL resection at $\mathrm{C6}$-C7 after TDA placement had no effect on either segmental lordosis or disc height, suggesting the PLL played a minor role in influencing these segmental parameters at this level.

Lauryssen et al. $^{4}$ performed a clinical feasibility study on 30 patients using the same cervical disc prosthesis as the current study. While handling of the PLL was not explicitly stated in the manuscript, personal communications with the authors verified that PLL was resected in all cases. The majority of implanted levels were at C5-C6 with a resulting change in segmental lordosis of 3.1 degrees and change in anterior and posterior disc height of 2.6 $\mathrm{mm}$ and $1.8 \mathrm{~mm}$, respectively. These results compare favorably with this study's C5-C6 change in segmental lordosis data $(+2.8 \pm 3.5$ degrees $)$ and disc height data (anterior height of $+2.2 \pm 0.7 \mathrm{~mm}$ and posterior height of $+1.6 \pm 1.0 \mathrm{~mm}$ ) in which the PLL was similarly resected at the time of TDA implantation.

In contrast to previous publications, ${ }^{12,16,24}$ the methodology used in the current study maintained continuity of the experiment before and after C6-C7 PLL resection by maintaining TDA position and minimizing alteration to other structural tissues.

The difference between previous studies investigating TDA ROM and stiffness ${ }^{12,35}$ and the current results may be related to the difference in the design of artificial discs used. The literature shows that 2level TDA with PLL resection can show additive rotational instability, ${ }^{16,19}$ suggesting the importance of retaining critical support structures such as PLL or compensation through prosthetic devices. In contrast, ROM over the C5-C7 segments in our study decreased for both the 1- and the 2-level TDAs with respect to intact, supporting the notion that the choice of implant design may have a
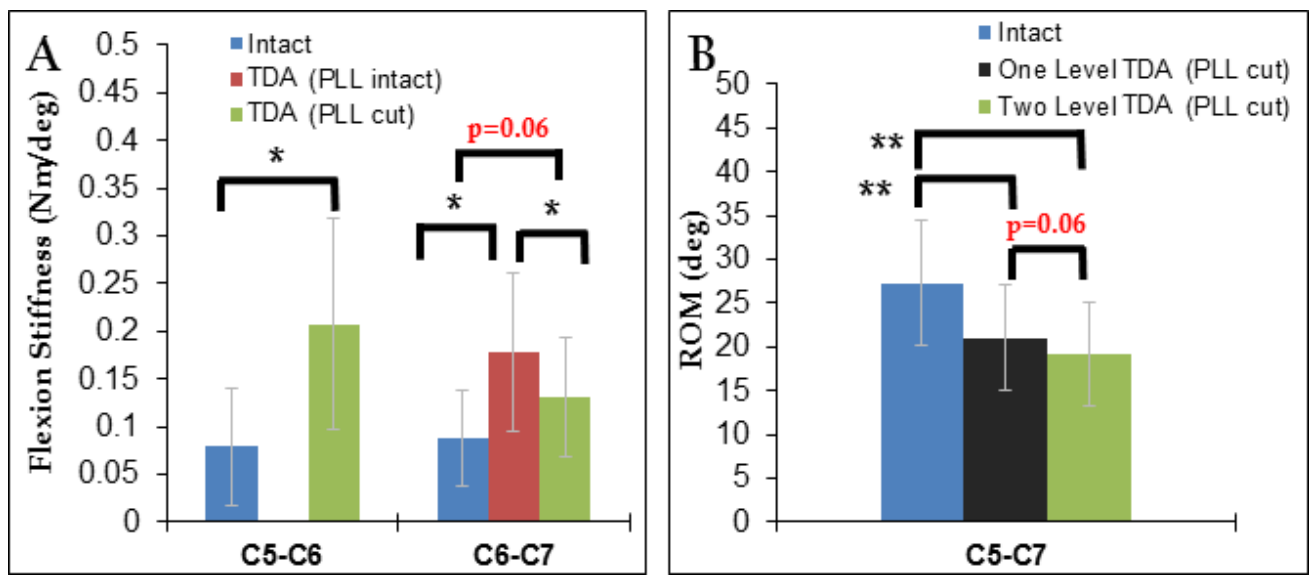

Figure 7. (A) Flexion stiffness of $\mathrm{C} 5-\mathrm{C} 6$ and $\mathrm{C} 6-\mathrm{C} 7$ for relevant protocol steps. (B) $\mathrm{C} 5-\mathrm{C} 7$ flexion-extension range of motion (ROM) of intact, 1-level total disc arthroplasty (TDA) (posterior longitudinal ligament [PLL] resection) and 2-level TDA (PLL resection). ${ }^{*} P \leq .05,{ }^{\star *} P \leq .01$. 
significant impact on motion segmental stability. 24,33

The current study has some limitations. It was performed on relatively healthy specimens with no significant disc degeneration in order to eliminate confounding factors, such as bridging osteophytes, facet and disc degeneration, and so on. The results may be valid in the clinical setting if the surgical technique is similar to those described in this study, (narrow window, minimal bone removal, and decompression). Implants with 6-mm height were used at all levels. As a result, the average disc height after TDA increased about $1.5 \mathrm{~mm}(1.9 \mathrm{~mm}$ at C5C6, $1.1 \mathrm{~mm}$ at C6-C7) beyond the intact segmental height. This increased height could cause recruitment of remaining soft tissue structures, adding to the increase in segmental stiffness. It is complex to determine the contribution of this ligament tensioning ("ligamentotaxis"). The change in stiffness after TDA was a combination of TDA stiffness, PLL stiffness, and increased soft tissue tension caused by increased segmental height. At C6-C7 with the PLL cut, $56 \%$ of this change in stiffness was recovered, leaving the remainder to be distributed between the TDA and soft tissues. Further analysis of increase in height can be performed if devices with smaller heights become available. Finally, the effect of PLL resection was investigated with 1 design of cervical disc prosthesis. Additional research is needed to assess motion segment kinematics before and after PLL resection in disc arthroplasty using other prosthesis designs.

\section{CONCLUSION}

Resection of the PLL did not significantly affect motion segment kinematics after 1-level cervical TDA using a prosthesis with inherent stiffness properties. Its removal increased flexion ROM without degrading stiffness below intact values. The 2-level model also remained more stable than intact after arthroplasty with PLL resection at both levels. Motion segment stiffness loss after PLL resection can be compensated for by a TDA design that can provide resistance to angular motion.

\section{REFERENCES}

1. Miao J, Yu F, Shen Y, et al. Clinical and radiographic outcomes of cervical disc replacement with a new prosthesis. Spine J. 2014;14(6):878-883.

2. Yang DL, Ding WY, Zhang YZ, et al. Removal versus preservation of the posterior longitudinal ligament in Bryan cervical disc arthroplasty. Chin Med J (Engl). 2013;126(20):38123816.

3. Coric D, Finger F, Boltes P. Prospective randomized controlled study of the Bryan Cervical Disc: early clinical results from a single investigational site. J Neurosurg Spine. 2006;4(1):31-35.

4. Lauryssen C, Coric D, Dimmig T, et al. Cervical total disc replacement using a novel compressible prosthesis: results from a prospective Food and Drug Administration-regulated feasibility study with 24-month follow-up. Int J Spine Surg. 2012;6:71-77.

5. Vital JM, Boissiere L, Obeid I. C6-C7 cervical disc arthroplasty in cervical disc herniation. Eur Spine $J$. 2013;22(9):2136-2138.

6. Humphreys SC, Hodges SD, Fisher DL, et al. Reliability of magnetic resonance imaging in predicting disc material posterior to the posterior longitudinal ligament in the cervical spine. A prospective study. Spine (Phila Pa 1976). 1998;23(22):2468-2471.

7. Wang X, Chen Y, Chen D, et al. Removal of posterior longitudinal ligament in anterior decompression for cervical spondylotic myelopathy. J Spinal Disord Tech. 2009;22(6):404407.

8. Ulrich C, Woersdoerfer O, Kalff R, et al. Biomechanics of fixation systems to the cervical spine. Spine (Phila Pa 1976). 1991;16(suppl 3):S4-S9.

9. Teo EC, Ng HW. Evaluation of the role of ligaments, facets and disc nucleus in lower cervical spine under compression and sagittal moments using finite element method. Med Eng Phys. 2001;23(3):155-164.

10. Goel VK, Clausen JD. Prediction of load sharing among spinal components of a C5-C6 motion segment using the finite element approach. Spine (Phila Pa 1976). 1998;23(6):684691.

11. Loughenbury PR, Wadhwani S, Soames RW. The posterior longitudinal ligament and peridural (epidural) membrane. Clin Anat. 2006;19(6):487-492.

12. McAfee PC, Cunningham B, Dmitriev A, et al. Cervical disc replacement-porous coated motion prosthesis: a comparative biomechanical analysis showing the key role of the posterior longitudinal ligament. Spine (Phila Pa 1976). 2003;28(20):S176-S185.

13. Akaishi F. [Biomechanical properties of the anterior and posterior longitudinal ligament in the cervical spine]. Nihon Ika Daigaku Zasshi. 1995;62(4):360-368.

14. Chin KR, Ghiselli G, Cumming V, et al. Postoperative magnetic resonance imaging assessment for potential compressive effects of retained posterior longitudinal ligament after anterior cervical fusions: a cross-sectional study. Spine (Phila Pa 1976). 2013;38(3):253-256.

15. Tkaczuk H. Tensile properties of human lumbar longitudinal ligaments. Acta Orthop Scand. 1968;(suppl 115):1+.

16. McAfee PC, Cunningham BW, Hayes V, et al. Biomechanical analysis of rotational motions after disc arthroplasty: implications for patients with adult deformities. Spine (Phila Pa 1976). 2006;31(suppl 19):S152-S160.

17. Chen TY, Crawford NR, Sonntag VK, et al. Biomechanical effects of progressive anterior cervical decompression. Spine (Phila Pa 1976). 2001;26(1):6-13; discussion 4.

18. Panjabi MM, White AA III, Johnson RM. Cervical 
spine mechanics as a function of transection of components. $J$ Biomech. 1975;8(5):327-336.

19. Roberto RF, McDonald T, Curtiss S, et al. Kinematics of progressive circumferential ligament resection (decompression) in conjunction with cervical disc arthroplasty in a spondylotic spine model. Spine (Phila Pa 1976). 2010;35(18):1676-1683.

20. Cunningham BW, Hu N, Zorn CM, et al. Biomechanical comparison of single- and two-level cervical arthroplasty versus arthrodesis: effect on adjacent-level spinal kinematics. Spine J. 2010;10(4):341-349.

21. Goel VK, Faizan A, Palepu V, et al. Parameters that effect spine biomechanics following cervical disc replacement. Eur Spine J. 2012;21(suppl 5):S688-S699.

22. Jaramillo-de la Torre JJ, Grauer JN, Yue JJ. Update on cervical disc arthroplasty: where are we and where are we going? Curr Rev Musculoskelet Med. 2008;1(2):124-130.

23. Yoganandan N, Pintar F, Butler J, et al. Dynamic response of human cervical spine ligaments. Spine (Phila Pa 1976). 1989;14(10):1102-1110.

24. Cakir B, Richter M, Schmoelz W, et al. Resect or not to resect: the role of posterior longitudinal ligament in lumbar total disc replacement. Eur Spine J. 2012;21(suppl 5):S592S598.

25. Patwardhan AG, Havey RM, Carandang G, et al. Effect of compressive follower preload on the flexion-extension response of the human lumbar spine. J Orthop Res. 2003;21(3):540-546.

26. Patwardhan AG, Havey RM, Ghanayem AJ, et al. Load-carrying capacity of the human cervical spine in compression is increased under a follower load. Spine (Phila Pa 1976). 2000;25(12):1548-1554.

27. Havey RM, Goodsitt J, Khayatzadeh S, et al. Threedimensional computed tomography-based specimen-specific kinematic model for ex vivo assessment of lumbar neuroforaminal space. Spine (Phila Pa 1976). 2015;40(14):E814E822.

28. Holm S. Sequentially rejective multiple test procedure. Scand. J. Stat. 1979;6(2):65-70.

29. Durbhakula MM, Ghiselli G. Cervical total disc replacement, part I: rationale, biomechanics, and implant types. Orthop Clin North Am. 2005;36(3):349-354.

30. Bertagnoli R, Kumar S. Indications for full prosthetic disc arthroplasty: a correlation of clinical outcome against a variety of indications. Eur Spine J. 2002;11(suppl 2):S131-S136.

31. Sears WR, Duggal N, Sekhon LH, et al. Segmental malalignment with the Bryan cervical disc prosthesis - contributing factors. J Spinal Disord Tech. 2007;20(2):111-117.

32. Yoon DH, Yi S, Shin HC, et al. Clinical and radiological results following cervical arthroplasty. Acta Neurochir (Wien). 2006;148(9):943-950.

33. Buchowski JM, Anderson PA, Sekhon L, et al. Cervical disc arthroplasty compared with arthrodesis for the treatment of myelopathy. Surgical technique. J Bone Joint Surg Am. 2009;91(suppl 2):223-232.

34. Murrey D, Janssen M, Delamarter R, et al. Results of the prospective, randomized, controlled multicenter Food and Drug Administration investigational device exemption study of the ProDisc-C total disc replacement versus anterior discectomy and fusion for the treatment of 1-level symptomatic cervical disc disease. Spine J. 2009;9(4):275-286.

35. Puttlitz CM, DiAngelo DJ. Cervical spine arthroplasty biomechanics. Neurosurg Clin N Am. 2005;16(4):589-594.

Disclosures and COI: This study was supported by funds from the Department of Veterans Affairs, Washington, DC, and Spinal Kinetics Inc, Sunnyvale, CA.

Corresponding Author: Leonard I. Voronov, MD, PhD, Department of Orthopaedic Surgery and Rehabilitation, Loyola University Chicago, 2160 South First Avenue, Maywood, IL 60153, Phone: (708) 202-5802, Fax: (708) 202-7938, Email: 1voronov@hotmail.com.

Published 3 August 2018

This manuscript is generously published free of charge by ISASS, the International Society for the Advancement of Spine Surgery. Copyright (C) 2018 ISASS. To see more or order reprints or permissions, see http://ijssurgery.com. 